\title{
Lindner, Konstantin / Zimmermann, Mirjam (202 I). Handbuch ethische Bildung. Religionspädagogische Fokussierungen. Tübingen: Mohr Siebeck. ISBN 978-3-8252-5604-3. 388 Seiten.
}

\section{Simone Horstmann}

Technische Universität Dortmund (simone.horstmann@tu-dortmund.de)

Wann immer Ethik nicht allein als theoretische Reflexion von Moral, sondern darüber hinaus als umfassender Bildungsanspruch konzeptualisiert wird, erwächst aus der Multidimensionalität dieser Verortung ein nicht unbeträchtlicher Komplexitätszuwachs: Ethische Bildung hat dann Bildungssubjekte, d. h. Lernende ebenso wie Lehrende, psychologische, aber auch institutionelle, religionsplurale, historische und rechtliche Voraussetzungshaftigkeiten und Kontexte zu berücksichtigen. Erschwerend dürfte hinzukommen, dass auch diese Bedingungsstrukturen nicht singulär erfasst werden können, sondern sich gegenseitig beeinflussen (können). Der von Konstantin Lindner und Mirjam Zimmermann herausgegebene Band bildet diese Komplexität in überzeugender Weise ab und thematisiert sie vor dem Hintergrund einer religionspädagogischen Perspektive; er repräsentiert damit gewissermaßen das breite Anspruchsportfolio ethischer Bildung, dem (universitär wie schulisch) Lehrende theoretisch wie praktisch begegnen. Und obschon die Herausgeber*innen das Handbuch in die historische Kontinuität und die Traditionslinie vorheriger Lehrbuch-Publikationen stellen (4f.), setzt der Band eigene und sehr beachtenswerte Schwerpunkte, die hier angesprochen werden sollen.

Ein erstes Großkapitel des Handbuchs räumt zunächst den Adressat*innen ethischer Bildung einen zentralen Ort ein: Dazu thematisieren vier Beiträge die ethisch relevanten lebensweltlichen Fragen von Kindern und Jugendlichen, deren empirisch fassbare Wertorientierungen und kognitive bzw. emotionale Entwicklung ebenso wie die Bereiche Familie, Freunde, Schule und Religion als Einflussfaktoren auf die ethische Urteilsbildung. Bereits hier fällt zudem eine Einschätzung auf, die den gesamten Band prägt: Die Beiträge problematisieren die - lange Zeit selbstverständlich vorausgesetzte - Assoziation von Religion und Ethik bzw. ethischer Bildung in einem angemessen kritischen Sinne, wenn etwa Ulrich Riegel die empirische Diagnose äußert, dass der Einfluss von Religion „auf der Ebene individuellen Verhaltens [...] bei der überwiegenden Mehrheit der Kinder und Jugendlichen jedoch nur eine unterschwellige Rolle spielen dürfte“ (22).

Im zweiten und dritten Großkapitel werden fachwissenschaftliche Grundlagen vertieft, indem sowohl zentrale Begriffe wie auch ethische Begründungstypologien (deontologisch, utilitaristisch, tugendethisch, diskursethisch und narrativ) referiert werden. Daran anschließend werden zentrale Themen ethischer Bildung (bspw. Politische Ethik, Wirtschaftsethik, Medien-, Medizin-, Umwelt- und Tierethik) aufgeschlüsselt und problematisiert. Auch hier fällt bisweilen die wohltuende Distanzierung gegenüber vormaligen Selbstverständlichkeiten auf: So könne etwa, wie Ruben Zimmermann anmerkt, die „Bedeutsamkeit der Bibel im aktuellen ethischen Diskurs nicht mehr selbstverständlich“ (89) vorausgesetzt werden. Es gelingt den Beiträgen aber gleichwohl, aus der Diagnose dieser Unselbstverständlichkeiten heraus angemessene Antworten zu entwickeln und dazu bspw. an die funktionale Multidimensionalität biblischer Ethik im Hinblick auf ihre normenbegründende und zugleich -kritisierende Rolle in ethischen Lernsettings zu erinnern. Bemerkenswert sind aber auch die Reibungspunkte der verschiedenen Beiträge dieses Kapitels: Während einerseits dem Begriff des Subjekts ein eigener Beitrag gewidmet wird, um den Freiheitsbegriff anthropologisch und damit auch bildungstheoretisch zu verankern, zeigt etwa der Beitrag zur Tierethik, wie prekär diese Kategorie zugleich ist, sofern sie zugleich einen starken Anthro- 
pozentrismus begründet. Eben diese Gleichzeitigkeit, mit der in diesem Band (und im laufenden Diskurs) beispielsweise der Begriff der Subjektivität einerseits als freiheitstheoretische conditio sine qua non ethischer Bildung herausgehoben und dann an anderer Stelle in seinen anthropozentrischen und speziesistischen Verstrickungen kritisch reflektiert wird, bildet der Band ab - allerdings leider ohne sie als solche zu problematisieren. Gerade in diesen beiden umfangreichen Kapiteln zeigt sich zudem, dass mitunter eine stärkere formale Vergleichbarkeit in den analytischen Zugängen und Frageperspektiven der einzelnen Beiträge hilfreich gewesen wäre: Denn während einige der Beiträge zumindest knapp die bildungstheoretische Bedeutung der jeweiligen ethischen Ausführungen thematisieren, beschränken sich andere Beiträge auf eine rein ethische Diskussion.

Im Folgekapitel zeigt sich dann ein weiteres Spezifikum des Bandes: Ethische Bildung wird hier interreligiös verstanden und daher sowohl aus christlicher wie auch aus jüdischer, muslimischer, hinduistischer und buddhistischer Perspektive thematisiert; die Beiträge selbst greifen die interreligiösen Herausforderungen des ethischen Lernens allerdings kaum auf und bleiben bei einer binnenreligiösen Perspektive. Eine wichtige Blickweitung ergibt sich dann aus dem Anschlusskapitel, das genauer auf die Dimensionen ethischer Bildung im Religionsunterricht eingeht: Man erfährt hier Wesentliches zur historischen wie auch systematischen Voraussetzungshaftigkeit einer kulturkonstitutiven wie auch -kritischen Funktion(alisierung) von religiösen Bildungsansprüchen; so plädiert etwa Henrik Simojoki dafür, den Reichweitenverzicht, den das Konzept ethischer Bildung gegenüber einer umfassend religiös-sittlichen Erziehung in Kauf nimmt, als einen "Gewinn an Fokus“ und als einen „bemerkenswerten Zuwachs an bereichsbezogener Expertise“ (220) zu begreifen. Die darauffolgenden Beiträge konkretisieren dabei gewissermaßen dieses Plädoyer und zeigen exemplarisch mögliche Optionen einer ausdifferenzierten Expertise von Lehrkräften auf, indem sie etwa die Frage nach der Kompetenzorientierung im Rahmen ethischer Bildung im RU aufgreifen, nach einer inklusions- und gendersensiblen Gestaltung ethischer Bildung fragen oder konstruktivistische Analysezugänge reflektieren. Konstantin Lindner fasst das Anliegen einer solchen professionsorientierten Perspektive zusammen, indem er betont, Professionalität im Kontext ethischer Bildung bedeute, „im Wissen um die eigenen Vorannahmen so zu unterrichten, dass die Schülerinnen und Schüler den ethischen Lern- zu ihrem Bildungsprozess machen und sich von Lehrkräfteeinschätzungen auch distanzieren können“ (264). Zwei weitere Kapitel befassen sich schließlich mit zentralen Lernwegen und Medien ethischer Bildung sowie mit deren nicht-schulischen Kontexten (KiTa, Jugendarbeit, Erwachsenenbildung).

Damit bildet der Band eine gleichermaßen umfassende wie zugleich sinnvoll fokussierte Auseinandersetzung mit den Formen und Normen ethischer Bildung, die zudem sowohl konfessionelle wie fachliche Divergenzen abbildet; der Band ist im besten Sinne geeignet, um eine Professionalisierung ethischer Bildung insbesondere im Religionsunterricht anzuregen und zu begleiten. 